PROCEEDINGS OF THE

AMERICAN MATHEMATICAL SOCIETY

Volume 133, Number 1, Pages 175-184

S 0002-9939(04)07494-5

Article electronically published on July 26, 2004

\title{
CONVOLUTION OPERATORS INDUCED BY APPROXIMATE IDENTITIES AND POINTWISE CONVERGENCE IN $L_{p}(\mathbb{R})$ SPACES
}

\author{
PARTHENA AVRAMIDOU
}

(Communicated by Andreas Seeger)

\begin{abstract}
Given a sequence of kernels $\phi_{n}$ for which the operators $T_{n} f=$ $\phi_{n} * f$ converge a.e. in all $L_{p}(\mathbb{R})$ spaces, $p \geq 1$, a perturbation method is provided with the property that the modified convolution operators converge pointwise only in selective spaces.
\end{abstract}

\section{INTRODUCTION}

Nagel and Stein 3 showed the potential of more general than nontangential differentiation simultaneously in various $L_{p}\left(\mathbb{R}^{n}\right)$ spaces. They obtained a necessary and sufficient condition for the approach regions that determines boundedness of the associated Hardy-Littlewood type maximal operator in all $L_{p}\left(\mathbb{R}^{n}\right)$ spaces, $p \geq 1$. As an application, for kernels of the form $\phi_{n}=\frac{1}{\left|I_{n}\right|} \chi_{I_{n}}$, where $\left\{I_{n}\right\}$ is a sequence of shrinking intervals approaching the origin, the corresponding convolution operators either converge pointwise in all $L_{p}\left(\mathbb{R}^{n}\right)$ spaces, $p \geq 1$, or in none.

In the ergodic averages setting, Bellow [1] and Reinhold-Larsson [4] constructed examples of sequences of natural numbers along which the individual ergodic theorem holds in some $L_{p}$ spaces (good behavior) and not in others (bad behavior). In particular, well-behaved sequences were perturbed in such a way that good behavior persists only in certain spaces. A key role in the constructions was played by a stability condition of Emerson [2]. One can prove that moving averages display the same behavior.

The present work illustrates the same possibility of discrepancy in differentiation in $L_{p}(\mathbb{R})$ spaces. We provide a method for restricting selectively the pointwise convergence of convolution operators for a wide class of kernel functions, which we call approximate identities.

This paper comprises part of the author's thesis. She would like to acknowledge the invaluable guidance of her advisor, Professor Joseph Rosenblatt, and the helpful comments and advice of Professor Roger Jones.

Received by the editors August 6, 2003 and, in revised form, September 11, 2003.

2000 Mathematics Subject Classification. Primary 28A15, 42A85; Secondary 43A15.

(C)2004 American Mathematical Society Reverts to public domain 28 years from publication 


\section{Preliminaries}

In this section we state a variant of Banach's Principle, we introduce the necessary terminology, and we prove some auxiliary propositions.

It is well known that the pointwise behavior of a sequence of operators is closely related to the behavior of its maximal operator. Banach's Principle relates the finiteness a.e. and the continuity in measure of the maximal operator. Under some extra assumptions, Stein [6] connected the finiteness a.e. and the type of the maximal operator in $L_{p}$ spaces for $1 \leq p \leq 2$. Finally, Sawyer 5 ] added the assumption of positivity and treated all $L_{p}$ spaces, $p \geq 1$. The quantitative character of Sawyer's Principle makes it more applicable. The setting needed is the following:

(a) $(\Omega, \mathcal{F}, \mu)$ is a probability measure space.

(b) $\left\{T_{k}\right\}$ is a sequence of linear, continuous in measure operators from some $L_{p}(\Omega), 1 \leq p \leq+\infty$, to $\mathcal{M}(\Omega)$, the set of all $\mu$-measurable finite a.e. functions, and $T^{*}$ is the maximal operator.

(c) Each $T_{k}$ is positive.

(d) There is a family of mappings $\left(S_{\alpha}\right)_{\alpha \in I}$ from $\Omega$ to $\Omega$ that are measure preserving, and mixing in the following sense:

if $A, B \in \mathcal{F}$ and $\rho>1$, then there exists $S_{\alpha}$ such that

$$
\mu\left(A \cap S_{\alpha}^{-1}(B)\right) \leq \rho \mu(A) \mu(B) .
$$

(e) $T^{*}$ and $\left(S_{\alpha}\right)_{\alpha \in I}$ commute in the following sense:

for each $S_{\alpha}, T^{*}\left(S_{\alpha} f\right)(x) \geq S_{\alpha}\left(T^{*} f\right)(x)$ for all $f \in L_{p}(\Omega), x \in \Omega$.

Theorem 2.1 ([5]). Let $(\Omega, \mathcal{F}, \mu),\left\{T_{k}\right\}$ and $\left(S_{\alpha}\right)_{\alpha \in I}$ be as above. Then $T^{*}$ is of weak type $(p, p)$ if and only if for each $f \in L_{p}(\Omega), T^{*} f(x)<+\infty$ a.e.

We consider convolution operators with kernel functions of a special type that we call approximate identities.

Definition 2.2. A sequence of functions $\left\{\phi_{n}\right\}_{n \in \mathbb{N}}$ is called an approximate identity if $\phi_{n} \geq 0, \int_{\mathbb{R}} \phi_{n}=1$ and for every $f \in L_{1}(\mathbb{R}), \lim _{n \rightarrow \infty}\left\|\phi_{n} * f-f\right\|_{1}=0$.

Remark 2.3. Convex combinations of approximate identities form new approximate identities.

The next proposition gives an equivalent characterization of approximate identities.

Proposition 2.4. A sequence of functions $\left\{\phi_{n}\right\}_{n \in \mathbb{N}}$ with $\phi_{n} \geq 0, \int_{\mathbb{R}} \phi_{n}=1$ is an approximate identity if and only if for every $\varepsilon>0$ there exists $n_{0} \in \mathbb{N}$ so that for all $n \geq n_{0}$ we have $\int_{-\varepsilon}^{\varepsilon} \phi_{n}>1-\varepsilon$.

Proof. Suppose that $\left\{\phi_{n}\right\}$ is an approximate identity, and that there exists $\varepsilon>0$ so that for every $n \in \mathbb{N}$ there exists $m \geq n$ such that

$$
\int_{-\varepsilon}^{\varepsilon} \phi_{m} \leq 1-\varepsilon\left(\Leftrightarrow \int_{\{|x|>\varepsilon\}} \phi_{m}(x) d x \geq \varepsilon\right) .
$$

Let $f(x)=\chi_{\{|x|<\varepsilon / 2\}}(x) \in L_{1}(\mathbb{R})$. For $\tilde{\varepsilon}=\varepsilon^{2}$ there exists $n_{0}$ so that for all $n \geq n_{0}$ we have $\left\|\phi_{n} * f-f\right\|_{1}<\tilde{\varepsilon}$. On the other hand, there exists $m \geq n_{0}$ so that

$$
\int_{\{|x|>\varepsilon\}} \phi_{m}(x) d x \geq \varepsilon \text {. }
$$


Applying Fubini's theorem yields

$$
\begin{aligned}
\left\|\phi_{m} * f-f\right\|_{1} & \geq \int_{\{|x|>\varepsilon / 2\}} \int_{-\varepsilon / 2}^{\varepsilon / 2} \phi_{m}(x-y) d y d x \\
& \geq \int_{-\varepsilon / 2}^{\varepsilon / 2} \int_{\{|t|>\varepsilon\}} \phi_{m}(t) d t d y \geq \tilde{\varepsilon} .
\end{aligned}
$$

By contradiction, we obtain the wanted conclusion.

Conversely, let $\mathfrak{B}$ denote the Borel measurable functions. For $g \in \mathfrak{B}$ we have that $g(x-y)$ is measurable in $\mathbb{R}^{2}$. Therefore, for any approximate identity $\left\{\phi_{n}\right\}$ we can define $\phi_{n} * g$. For every $f \in L_{1}(\mathbb{R})$ there exists $g \in \mathfrak{B}$ such that $f=g$ a.e. Moreover, for all $g_{1}, g_{2}$ in the same equivalence class, $\phi_{n} * g_{1}(x)=\phi_{n} * g_{2}(x)$ a.e. Hence, for $f \in L_{1}(\mathbb{R})$ we can define $\phi_{n} * f$ a.e. Now consider

$$
\phi_{n} * f(x)-f(x)=\int_{\mathbb{R}} \phi_{n}(y)[f(x-y)-f(x)] d y,
$$

and set

$$
F_{n}(x, y)=\phi_{n}(y)[f(x-y)-f(x)] .
$$

Applying Minkowski's inequality for integrals we have

$$
\begin{aligned}
\left\|\int_{\mathbb{R}} F_{n}(\cdot, y) d y\right\|_{1} & \leq \int_{\mathbb{R}}\left\|F_{n}(\cdot, y)\right\|_{1} d y \\
& =\int_{\mathbb{R}} \phi_{n}(y)\|f(\cdot-y)-f(\cdot)\|_{1} d y,
\end{aligned}
$$

and the last term converges to 0 as $n \rightarrow \infty$ by the Lebesgue Dominated Convergence Theorem.

Proposition 2.5. If $\left\{\phi_{n}\right\}$ is an approximate identity, then for every $f \in L_{p}(\mathbb{R})$, $1 \leq p<+\infty, \lim _{n \rightarrow \infty}\left\|\phi_{n} * f-f\right\|_{p}=0$.

Proof. This follows by the same argument as the converse direction in the last proposition, but using the $L_{p}$-norm rather than the $L_{1}$-norm.

The following proposition provides a stability criterion that takes the place of Emerson's result.

Proposition 2.6. Let $\phi_{n}=\alpha_{n} \psi_{n}+\left(1-\alpha_{n}\right) \sigma_{n}$, where $\left\{\psi_{n}\right\}_{n \in \mathbb{N}},\left\{\sigma_{n}\right\}_{n \in \mathbb{N}}$ are approximate identities and $0 \leq \alpha_{n} \leq 1$.

(a) For $1 \leq p<+\infty$ and every $f \in L_{p}(\mathbb{R}), \lim _{n \rightarrow \infty}\left\|\left(\phi_{n}-\psi_{n}\right) * f\right\|_{p}=0$ and $\lim _{n \rightarrow \infty}\left\|\left(\phi_{n}-\sigma_{n}\right) * f\right\|_{p}=0$.

(b) For every $f \in L_{\infty}(\mathbb{R}), \lim _{n \rightarrow \infty}\left(\phi_{n}-\psi_{n}\right) * f(x)=0$ a.e.

(c) For $1 \leq p<+\infty$, if $\sum_{n}\left(1-\alpha_{n}\right)^{p}<+\infty$, then for every $f \in L_{p}(\mathbb{R})$, $\lim _{n \rightarrow \infty}\left(\phi_{n}-\psi_{n}\right) * f(x)=0$ a.e.

Proof. (a) Fix $1 \leq p \leq+\infty$, and let $f \in L_{p}(\mathbb{R})$. By applying Minkowski's inequality,

$$
\left\|\left(\phi_{n}-\psi_{n}\right) * f\right\|_{p} \leq\left(1-\alpha_{n}\right)\left(\left\|\sigma_{n} * f-f\right\|_{p}+\left\|\psi_{n} * f-f\right\|_{p}\right),
$$

and, since both $\left\{\psi_{n}\right\}$ and $\left\{\sigma_{n}\right\}$ are approximate identities, $\left\|\left(\phi_{n}-\psi_{n}\right) * f\right\|_{p} \rightarrow 0$. $\left\|\left(\phi_{n}-\sigma_{n}\right) * f\right\|_{p} \rightarrow 0$ follows similarly.

(b) For $f \in L_{\infty}(\mathbb{R}),\left|\left(\phi_{n}-\psi_{n}\right) * f(x)\right| \leq\left\|\left(\phi_{n}-\psi_{n}\right) * f\right\|_{\infty}$ and the last term tends to zero by part (a). 
(c) For $f \in L_{p}(\mathbb{R})$,

$$
\begin{aligned}
\int \sum_{n}\left(1-\alpha_{n}\right)^{p}\left|\sigma_{n} * f(x)\right|^{p} d x & =\sum_{n}\left\|\left(1-\alpha_{n}\right) \sigma_{n} * f\right\|_{p}^{p} \\
& \leq \sum_{n}\left(1-\alpha_{n}\right)^{p}\left\|\sigma_{n}\right\|_{1}^{p}\|f\|_{p}^{p}<+\infty .
\end{aligned}
$$

Then $\left(1-\alpha_{n}\right) \sigma_{n} * f(x) \rightarrow 0$ a.e. Similarly, $\left(\alpha_{n}-1\right) \psi_{n} * f(x) \rightarrow 0$ a.e.

Definition 2.7. An approximate identity $\left\{\phi_{n}\right\}$ is called $L_{p}$ good if $\phi_{n} * f \rightarrow f$ a.e. for all $f \in L_{p}(\mathbb{R})$, and it is called $\operatorname{good}$ if it is $L_{p}$-good for every $1 \leq p \leq+\infty$. An approximate identity $\left\{\phi_{n}\right\}$ is called $L_{p}$-bad if there exists $f \in L_{p}(\mathbb{R})$ such that $\phi_{n} * f \not f$ on a set of positive measure.

Combining the last definition and proposition we conclude the following.

Corollary 2.8. If $\left\{\psi_{n}\right\}_{n \in \mathbb{N}}$ is an $L_{\infty}$-good approximate identity, $\left\{\sigma_{n}\right\}_{n \in \mathbb{N}}$ is any approximate identity and $\phi_{n}=\alpha_{n} \psi_{n}+\left(1-\alpha_{n}\right) \sigma_{n}$ with $0 \leq \alpha_{n} \leq 1$ and $\alpha_{n} \rightarrow 1$, then $\left\{\phi_{n}\right\}_{n \in \mathbb{N}}$ is also an $L_{\infty}$-good approximate identity. If $\left\{\psi_{n}\right\}_{n \in \mathbb{N}}$ is an $L_{p}$-good approximate identity for some $1 \leq p<+\infty$ and $\sum_{n}\left(1-\alpha_{n}\right)^{p}<+\infty$, then $\left\{\phi_{n}\right\}_{n \in \mathbb{N}}$ is also an $L_{p}$-good approximate identity.

Definition 2.9. Let $\left\{\psi_{n}\right\}_{n \in \mathbb{N}}$ and $\left\{\sigma_{n}\right\}_{n \in \mathbb{N}}$ be approximate identities, $\alpha_{n}$ be a sequence of real numbers with $0 \leq \alpha_{n} \leq 1$ and $\alpha_{n} \rightarrow 1$. We call perturbed approximate identity any approximate identity $\left\{\phi_{n}\right\}_{n \in \mathbb{N}}$ of the form $\phi_{n}=\alpha_{n} \psi_{n}+\left(1-\alpha_{n}\right) \sigma_{n}$.

Remark 2.10. The above definition provides a method of changing or "perturbing" an approximate identity $\left\{\psi_{n}\right\}$ by convex combinations with another approximate identity $\left\{\sigma_{n}\right\}$. The weights are chosen appropriately so that the resulting approximate identity will not differ dramatically from the original one.

\section{MAin RESUlt}

Theorem 3.1. (1) For fixed $p \in[1, \infty)$,

(a) given any good approximate identity $\left\{\psi_{n}\right\}_{n \in \mathbb{N}}$ there exists a perturbed approximate identity $\left\{\phi_{n}\right\}_{n \in \mathbb{N}}$ that is $L_{q}$-good for all $q \geq p$, and $L_{q}$-bad for all $1 \leq q<p$

(b) given any good approximate identity $\left\{\psi_{n}\right\}_{n \in \mathbb{N}}$ there exists a perturbed approximate identity $\left\{\phi_{n}\right\}_{n \in \mathbb{N}}$ that is $L_{q}$-good for all $q>p$, and $L_{q}$-bad for all $1 \leq q \leq p$.

(2) Given any good approximate identity $\left\{\psi_{n}\right\}_{n \in \mathbb{N}}$ there exists a perturbed approximate identity $\left\{\phi_{n}\right\}_{n \in \mathbb{N}}$ that is $L_{\infty}$-good, and $L_{q^{-}}$bad for all $1 \leq q<+\infty$.

Proof. (1a). For $p=1$ no perturbation is needed. Henceforth, assume that $p>1$. Let $\left\{\psi_{n}\right\}_{n \in \mathbb{N}}$ be a good approximate identity, and let $\left\{\zeta_{n}\right\}_{n \in \mathbb{N}}$ be any approximate identity. We modify $\left\{\zeta_{n}\right\}$ by setting $\sigma_{n}(\cdot)=\zeta_{m_{n}}\left(-t_{n}+\cdot\right)$ where $t_{n} \searrow 0$ and $m_{n} \nearrow \infty$ are to be chosen later. Let

$$
\phi_{n}=\alpha_{n} \psi_{n}+\left(1-\alpha_{n}\right) \sigma_{n},
$$

where

$$
1-\alpha_{n}=\frac{1}{\left(n \log ^{2} n\right)^{1 / p}}
$$


Lemma 3.2. $\left\{\phi_{n}\right\}$ is an $L_{q}$-good approximate identity for all $q \geq p$.

Proof. Fix $q \geq p$. Since $\sum_{n}\left(1-\alpha_{n}\right)^{q}<+\infty$ and $\left\{\psi_{n}\right\}$ is an $L_{q}$-good approximate identity, Proposition 2.6 ascertains that $\left\{\phi_{n}\right\}$ is also an $L_{q}$-good approximate identity.

Let

$$
\begin{gathered}
r_{n}=\frac{1}{n^{1+\frac{1}{p}}(\log n)^{\frac{2}{p}}}, \\
a_{n}=r_{n}^{\frac{1}{p+1}}=\frac{1}{n^{\frac{1}{p}}(\log n)^{\frac{2}{p(p+1)}}},
\end{gathered}
$$

$J_{n}=\left[a_{n}-r_{n}, a_{n}+r_{n}\right]$, and $U_{n}=\left(-a_{n}+r_{n},-a_{n+1}+r_{n+1}\right]$.

Lemma 3.3. For all $x \in U_{n}$ and for all $t \in-J_{n}$,

$$
x-t \leq C r_{n}(\log n)^{\frac{2}{p+1}}
$$

for some constant $C=C(p)$.

Proof. Since for $x \in U_{n}$ and for $t \in-J_{n}$,

$$
\begin{aligned}
x-t & \leq-a_{n+1}+r_{n+1}+a_{n}+r_{n} \\
& \leq 2 r_{n}+\left(a_{n}-a_{n+1}\right),
\end{aligned}
$$

it is sufficient to show that

$$
a_{n}-a_{n+1} \leq C r_{n}(\log n)^{\frac{2}{p+1}} .
$$

Let

$$
f(x)=\frac{1}{x^{\frac{1}{p}}(\log x)^{\frac{2}{p(p+1)}}} .
$$

Then $f^{\prime}(x)<0$ and $f^{\prime \prime}(x)>0$. Therefore, $f(x)-f(x+1) \leq-f^{\prime}(x)$. Using $f(n)=a_{n}$ we have

$$
\begin{aligned}
a_{n}-a_{n+1} & \leq-f^{\prime}(n) \\
& \leq \frac{C}{n^{1+\frac{1}{p}}(\log n)^{\frac{2}{p(p+1)}}} \\
& =C r_{n}(\log n)^{\frac{2}{p+1}}
\end{aligned}
$$

Choose $t_{n}=-a_{n}$ and $m_{n}$ such that

$$
\int_{-J_{n}} \sigma_{n}>1-r_{n}>C
$$

for some constant $C$. This is possible because $\left\{\zeta_{n}\right\}$ is an approximate identity.

Lemma 3.4. For each $1 \leq q<p$ there exists $f_{q} \in L_{q}(\mathbb{R})$ so that $\lim \sup _{n}\left|\phi_{n} * f_{q}\right|=$ $+\infty$ on a set of positive measure.

Proof. Fix $1 \leq q<p$. Let

$$
f_{q}(x)=\frac{1}{\left(x \log ^{2}(x / 2)\right)^{\frac{1}{q}}} \chi_{(0,1]}(x) \in L_{q}(\mathbb{R}) .
$$


Fix $n$ sufficiently large. For all $k \geq n$ and for all $x \in U_{k}$,

$$
\begin{aligned}
\phi_{k} * f_{q}(x) & \geq\left(1-\alpha_{k}\right) \sigma_{k} * f_{q}(x) \\
& \geq \frac{1}{\left(k \log ^{2} k\right)^{\frac{1}{p}}} \int_{-J_{k}} \sigma_{k}(t) f_{q}(x-t) d t,
\end{aligned}
$$

and using Lemma 3.3 ,

$$
\phi_{k} * f_{q}(x) \geq \frac{f_{q}\left(C r_{k}(\log k)^{\frac{2}{p+1}}\right)}{\left(k \log ^{2} k\right)^{\frac{1}{p}}} \int_{-J_{k}} \sigma_{k}(t) d t .
$$

Notice that

$$
r_{k}(\log k)^{\frac{2}{p+1}}=\frac{1}{k^{1+\frac{1}{p}}(\log k)^{\frac{2}{p(p+1)}}},
$$

which gives

$$
f_{q}\left(C r_{k}(\log k)^{\frac{2}{p+1}}\right)=\frac{k^{\frac{1}{q}+\frac{1}{p q}}(\log k)^{\frac{2}{p q(p+1)}}}{C^{\frac{1}{q}}\left(\log \left(C /\left(2 k^{\frac{p+1}{p}}(\log k)^{\frac{2}{p(p+1)}}\right)\right)^{\frac{2}{q}}\right.} .
$$

Let

$$
g_{q}(k)=\frac{(\log k)^{\frac{2}{p q(p+1)}-\frac{2}{p}}}{C^{\frac{1}{q}}\left(\log \left(C /\left(2 k^{\frac{p+1}{p}}(\log k)^{\frac{2}{p(p+1)}}\right)\right)\right)^{\frac{2}{q}}} .
$$

Then, using equations (3.1) and (3.2) we have

$$
\phi_{k} * f_{q}(x) \geq C k^{\frac{1}{q}-\frac{1}{p}+\frac{1}{p q}} g_{q}(k)>k^{\delta} \geq n^{\delta},
$$

where $\delta$ satisfies

$$
0<\delta<\frac{1}{q}-\frac{1}{p}+\frac{1}{p q} .
$$

It follows that for all $k \geq n$,

$$
U_{k} \subseteq\left\{\sup _{i} \phi_{i} * f_{q}>n^{\delta}\right\},
$$

which yields

$$
\bigcup_{k=n}^{\infty} U_{k} \subseteq\left\{\sup _{i} \phi_{i} * f_{q}>n^{\delta}\right\}
$$

or

$$
\left(-a_{n}+r_{n}, 0\right) \subseteq\left\{\sup _{i} \phi_{i} * f_{q}>n^{\delta}\right\} .
$$

Hence,

$$
\begin{aligned}
\left(n^{\delta}\right)^{q}\left|\left\{\sup _{i} \phi_{i} * f_{q}>n^{\delta}\right\}\right| & \geq n^{\delta q}\left(a_{n}-r_{n}\right) \\
& \geq C n^{\delta q} a_{n} \\
& \geq n^{\beta} \rightarrow+\infty
\end{aligned}
$$

for some $\frac{1}{p}<\beta<\delta q$. We conclude that the maximal operator is not of weak type $(q, q)$ in $L_{q}([0,1))$. Since the irrational rotations of $[0,1)$ form a family of measurepreserving transformations of $[0,1)$ to itself that is mixing and commuting with the maximal operator, we can apply Sawyer's Principle (Theorem 2.1). That implies the existence of a function $f \in L_{q}([0,1)) \subseteq L_{q}(\mathbb{R})$ such that $\lim _{\sup _{n}}\left|\phi_{n} * f\right|=+\infty$ a.e. on a set of positive measure in $\mathbb{R}$. 
(1b). Let $\left\{\psi_{n}\right\}_{n \in \mathbb{N}}$ be a good approximate identity, and let $\left\{\zeta_{n}\right\}_{n \in \mathbb{N}}$ be any approximate identity. Let $\left\{p_{n}\right\}$ be a sequence of real numbers satisfying

$$
p_{1}>p_{2}>\ldots>p_{n}>\ldots \searrow p \text {. }
$$

For each $p_{i}$ we can construct a perturbation $\left\{\phi_{n}^{i}\right\}_{n}$ of $\left\{\psi_{n}\right\}$ that is $L_{q^{-}}$good for $q \geq p_{i}$, and $L_{q}$-bad for $1 \leq q<p_{i}$, as described in part (1a). In particular,

$$
\phi_{n}^{i}=\alpha_{n}^{i} \psi_{n}+\left(1-\alpha_{n}^{i}\right) \sigma_{n}^{i}
$$

where

$$
1-\alpha_{n}^{i}=\frac{1}{\left(n \log ^{2} n\right)^{1 / p_{i}}}
$$

and

$$
\sigma_{n}^{i}(\cdot)=\zeta_{m_{n}^{i}}\left(-t_{n}^{i}+\cdot\right)
$$

The sequences $\left\{m_{n}^{i}\right\}$ and $\left\{t_{n}^{i}\right\}$ are chosen as expected, namely

$$
t_{n}^{i}=-\frac{1}{n^{\frac{1}{p_{i}}}(\log n)^{\frac{2}{p_{i}\left(p_{i}+1\right)}}}=-a_{n}^{i}
$$

and $m_{n}^{i}$ such that

$$
\int_{-J_{n}^{i}} \sigma_{n}^{i}>1-r_{n}^{i}>C
$$

where

$$
r_{n}^{i}=\frac{1}{n^{1+\frac{1}{p_{i}}}(\log n)^{\frac{2}{p_{i}}}}
$$

and $J_{n}^{i}=\left[a_{n}^{i}-r_{n}^{i}, a_{n}^{i}+r_{n}^{i}\right]$. Consider the sequence of blocks $\left\{B_{k}\right\}_{k \in \mathbb{N}}$, where $B_{k}=\left\{\phi_{n_{k-1}+1}^{k}, \ldots, \phi_{n_{k}}^{k}\right\}$, and $\left\{n_{k}\right\}$ is a sequence of positive integers increasing to infinity to be determined later. Let $D_{k}=\left\{n_{k-1}+1, \ldots, n_{k}\right\}$, and let $\left\{\phi_{n}\right\}_{n}=\bigcup_{k} B_{k}$.

Lemma 3.5. $\left\{\phi_{n}\right\}$ is an $L_{q}$-good approximate identity for all $q>p$.

Proof. Fix $q>p$. There exists $n_{0} \in \mathbb{N}$ so that for all $n \geq n_{0}$ we have $p_{n}<q$. Notice that

$$
\begin{aligned}
\sum_{k=n_{0}}^{\infty} \sum_{n \in D_{k}}\left(1-\alpha_{n}^{k}\right)^{q} & \leq \sum_{k=n_{0}}^{\infty} \sum_{n \in D_{k}} \frac{1}{\left(n \log ^{2} n\right)^{q / p_{n_{0}}}} \\
& \leq \sum_{n}\left(\frac{1}{n \log ^{2} n}\right)^{q / p_{n_{0}}}<+\infty
\end{aligned}
$$

and Proposition 2.6 finishes the proof.

Lemma 3.6. There exists a sequence of positive integers $\left\{n_{k}\right\}$ increasing to infinity such that $\left\{\phi_{n}\right\}$ is an $L_{p}$-bad approximate identity.

Proof. Consider a sequence $\left\{C_{i}\right\}$ such that $C_{i} \rightarrow+\infty$ as $i \rightarrow+\infty$. Let $n_{0}=1$. Fix $C_{i}$. Set $C=2^{(i-1) p+1} C_{i}$. Since $\left\{\phi_{n}^{i}\right\}_{n}$ is $L_{q}$-bad for all $q<p_{i}$, it is also $L_{p}$-bad. In particular, there exists $f_{i} \in L_{p}^{+}([0,1))$ with $\left\|f_{i}\right\|_{p}=2^{1-i}$ and $\lambda_{i}>0$ such that

$$
\begin{aligned}
\left|\left\{\sup _{n>n_{i-1}} \phi_{n}^{i} * f_{i}(x)>\lambda_{i}\right\}\right| & >\frac{C\left\|f_{i}\right\|_{p}^{p}}{\lambda_{i}^{p}} \\
& =\frac{2 C_{i}}{\lambda_{i}^{p}} .
\end{aligned}
$$


Therefore, there exists $n_{i}>n_{i-1}$ so that

$$
\left|\left\{\sup _{n_{i-1}<n \leq n_{i}} \phi_{n}^{i} * f_{i}>\lambda_{i}\right\}\right|>\frac{C_{i}}{\lambda_{i}^{p}} .
$$

Let

$$
\tilde{f}=\sum_{i} f_{i}
$$

Then

$$
\|\tilde{f}\|_{p} \leq \sum_{i}\left\|f_{i}\right\|_{p} \leq 2 .
$$

Suppose that $\left\{\phi_{n}\right\}$ satisfies a weak $(p, p)$ inequality in $L_{p}([0,1))$. Then there exists $C_{0}>0$ such that for all $\lambda>0$ and for all $f \in L_{p}^{+}([0,1))$,

$$
\left|\left\{\sup _{n} \phi_{n}^{i} * f>\lambda\right\}\right| \leq \frac{C_{0}\|f\|_{p}^{p}}{\lambda^{p}} .
$$

Consider $\lambda=\lambda_{i}$ and $f=\tilde{f}$. Then

$$
\begin{aligned}
\left|\left\{\sup _{n} \phi_{n} * f>\lambda\right\}\right| & \leq \frac{C_{0}\|f\|_{p}^{p}}{\lambda^{p}} \\
& =\frac{2^{p} C_{0}}{\lambda^{p}} .
\end{aligned}
$$

On the other hand,

$$
\begin{aligned}
\left|\left\{\sup _{n} \phi_{n} * f>\lambda\right\}\right| & \geq\left|\left\{\sup _{n_{i-1}<n \leq n_{i}} \phi_{n}^{i} * f_{i}>\lambda_{i}\right\}\right| \\
& >\frac{C_{i}}{\lambda^{p}} .
\end{aligned}
$$

Equations (3.3) and (3.4) imply that

$$
C_{0}>\frac{C_{i}}{2^{p}}
$$

but $C_{i} \rightarrow+\infty$ as $i \rightarrow+\infty$. Hence, there is no such constant $C_{0}$ and we conclude that $\left\{\phi_{n}\right\}$ is $L_{p}([0,1))$-bad.

Since the spaces $L_{q}([0,1))$ are nested, $\left\{\phi_{n}\right\}$ is $L_{q}([0,1))$-bad for all $1 \leq q \leq p$. Therefore, such a choice of $\left\{n_{k}\right\}$ makes $\left\{\phi_{n}\right\} L_{q}(\mathbb{R})$-bad for all $1 \leq q \leq p$.

(2). Let $\left\{\psi_{n}\right\}_{n \in \mathbb{N}}$ be a good approximate identity, and let $\left\{\zeta_{n}\right\}_{n \in \mathbb{N}}$ be any approximate identity. Let $\left\{p_{n}\right\}$ be a sequence of real numbers satisfying

$$
1 \leq p_{1}<p_{2}<\ldots<p_{n} \nearrow \infty .
$$

Consider the blocks $\left\{B_{k}\right\}$, where each block $B_{k}$ is related to $p_{k}$, as in part (1b). For $i \in D_{k}$, let

$$
\phi_{i}=\alpha_{i}^{k} \psi_{i}^{k}+\left(1-\alpha_{i}^{k}\right) \sigma_{i}^{k} .
$$

We choose $\left\{n_{k}\right\}$ growing fast enough so that $\alpha_{i}^{k} \rightarrow 1$. Then, since $\left\{\psi_{n}\right\}$ is $L_{\infty}$-good,

$$
\psi_{n} * f \rightarrow f \text { a.e. for all } f \in L_{\infty}(\mathbb{R}),
$$

and consequently,

$$
\alpha_{i}^{k} \psi_{i}^{k} * f \rightarrow f \text { a.e. for all } f \in L_{\infty}(\mathbb{R}) .
$$

On the other hand, since $\sigma_{i}^{k} * f(x) \leq\|f\|_{\infty}$, we have

$$
\left(1-\alpha_{i}^{k}\right) \sigma_{i}^{k} * f \rightarrow 0 \text { a.e. for all } f \in L_{\infty}(\mathbb{R}) .
$$


Thus, $\phi_{n} * f \rightarrow f$ a.e. for all $f \in L_{\infty}(\mathbb{R})$. Consider the sequence $\left\{C_{n}\right\}$ with the restriction

$$
C_{n} 2^{-n \alpha} \rightarrow+\infty
$$

for every constant $\alpha>0$.

Suppose that we chose $n_{k-1}$. The approximate identity $\left\{\phi_{n}^{k}\right\}_{n}$ is $L_{p_{m}}$-bad for every $m \in\{1,2, \ldots, k\}$, since it is $L_{q}$-bad for every $1 \leq q \leq p_{k}$. Therefore, there exists $f_{m}^{k} \in L_{p_{m}}([0,1))$ with $\left\|f_{m}^{k}\right\|_{p_{m}}=2^{-k}, \lambda_{m}^{k}>0$ and $n_{m}^{k}>n_{k-1}$ so that

$$
\begin{aligned}
\left|\left\{\sup _{n_{k-1}<n \leq n_{m}^{k}} \phi_{n}^{k} * f_{m}^{k}>\lambda_{m}^{k}\right\}\right| & >\frac{C_{k}\left\|f_{m}^{k}\right\|_{p_{m}}^{p_{m}}}{\left(\lambda_{m}^{k}\right)^{p_{m}}} \\
& =\frac{C_{k}}{2^{k p_{m}}\left(\lambda_{m}^{k}\right)^{p_{m}}} .
\end{aligned}
$$

Let

$$
n_{k}=\max _{1 \leq m \leq k} n_{m}^{k}
$$

Lemma 3.7. $\left\{\phi_{n}\right\}$ is $L_{p_{k}}$-bad for all $k \in \mathbb{N}$.

Proof. Fix $p_{k_{0}}$. Let

$$
\tilde{f}=\sum_{k \geq k_{0}} f_{k_{0}}^{k}
$$

Then

Moreover,

$$
\|\tilde{f}\|_{p_{k_{0}}}<2
$$

$$
\begin{aligned}
\left|\left\{\sup _{n} \phi_{n} * \tilde{f}>\lambda_{k_{0}}^{k}\right\}\right| & \leq \frac{C\|\tilde{f}\|_{k_{k_{0}}}^{p_{k_{0}}}}{\left(\lambda_{k_{0}}^{k}\right)^{p_{k_{0}}}} \\
& \leq \frac{2^{p_{k_{0}}} C}{\left(\lambda_{k_{0}}^{k}\right)^{p_{k_{0}}}}
\end{aligned}
$$

Hence,

$$
\begin{aligned}
\left|\left\{\sup _{n} \phi_{n} * \tilde{f}>\lambda_{k_{0}}^{k}\right\}\right| & \geq\left|\left\{\sup _{n_{k-1}<n \leq n_{k}} \phi_{n}^{k} * f_{k_{0}}^{k}>\lambda_{k_{0}}^{k}\right\}\right| \\
& >\frac{C_{k}}{2^{k p_{k_{0}}}\left(\lambda_{k_{0}}^{k}\right)^{p_{k_{0}}}} .
\end{aligned}
$$

Equations (3.5) and (3.6) imply that

$$
C>\frac{C_{k}}{2^{p_{k_{0}}(k+1)}} \rightarrow+\infty .
$$

The Marcinkiewicz interpolation theorem gives that $\left\{\phi_{n}\right\}$ is $L_{q}([0,1))$-bad for all $1 \leq q<+\infty$. Again, from $L_{q}([0,1))$ we obtain the same result for $L_{q}(\mathbb{R})$.

Example 3.8. Let $I_{n}=\left[\frac{1}{n}, \frac{3}{n}\right]$. Nagel and Stein's theorem [3] assures that the associated maximal operator satisfies a weak $(q, q)$ inequality for all $1 \leq q \leq+\infty$. That, in turn, yields that the set

$$
\mathcal{A}=\left\{f \in L_{q}(\mathbb{R}): \lim _{n \rightarrow \infty} \frac{1}{\left|I_{n}\right|} \int_{I_{n}} f(x+t) d t=f(x) \text { a.e. }\right\}
$$


is a closed set. Since the simple functions with support of finite measure form a subset of $\mathcal{A}$, dense in $L_{q}(\mathbb{R})$, we conclude that $\mathcal{A}=L_{q}(\mathbb{R})$. Therefore, $\left\{\psi_{n}\right\}_{n \in \mathbb{N}}$ given by $\psi_{n}=\frac{1}{I_{n}} \chi_{I_{n}}$ is a good approximate identity. Next we "perturb" $\left\{\psi_{n}\right\}$ as follows: let $J_{n}=\left[a_{n}-r_{n}, a_{n}+r_{n}\right]$, where

$$
r_{n}=\frac{1}{n\left(n \log ^{2} n\right)^{1 / p}} \text { and } a_{n}=r_{n}^{\frac{1}{p+1}} .
$$

Set

$$
\phi_{n}=\frac{1}{\left|I_{n}\right|+\left|J_{n}\right|} \chi_{I_{n} \cup J_{n}}
$$

By Theorem 3.1, part (1a), $\left\{\phi_{n}\right\}_{n \in \mathbb{N}}$ is an $L_{q}$-good approximate identity for $q \geq p$ and an $L_{q}$-bad approximate identity for $1 \leq q<p$.

The method used in proving Theorem 3.1 can be used in further perturbations of approximate identities to further restrict the good behavior.

Corollary 3.9. Let $\left\{\psi_{n}\right\}$ be an approximate identity that is $L_{q}$-good for $q \geq p$ $(q>p)$ and $L_{q}$-bad for $1 \leq q<p(1 \leq q \leq p)$. For every $s>p$ there exists a perturbed approximate identity $\left\{\phi_{n}\right\}$ that is $L_{q}$-good for $q \geq s(q>s)$ and $L_{q}$-bad for $1 \leq q<s(1 \leq q \leq s)$.

\section{REFERENCES}

1. A. Bellow, Perturbation of a Sequence, Advances in Mathematics 78 (1989), 131-139. MR91f:28009

2. W. Emerson, The pointwise ergodic theorem for amenable groups, Amer. J. Math. 96 (1974), 472-487. MR 50:7403

3. A. Nagel and E. M. Stein, On certain maximal functions and approach regions, Advances in Mathematics 54 (1984), 83-106. MR86a:42026

4. K. Reinhold-Larsson, Discrepancy of behavior of perturbed sequences in $L^{p}$ spaces, Proc. Amer. Math. Soc. 120 (1994), 865-874. MR94e:28008

5. S. Sawyer, Maximal inequalities of weak type, Ann. of Math. (2) 84 (1966), 157-174. MR 35:763

6. E. M. Stein, On limits of sequences of operators, Ann. of Math. (2) 74 (1961), 140-170. MR23:A2695

Department of Mathematics, Ohio State University, Columbus, Ohio 43210

E-mail address: avramidou@math.ohio-state.edu 